\title{
Estimating and Forecasting Volatility of Financial Time Series in Pakistan with GARCH-type Models
}

\author{
G.R. Pasha*, Tahira Qasim ${ }^{* *}$ and Muhammad Aslam
}

\section{Abstract}

In this paper we compare the performance of different GARCH models such as GARCH, EGARCH, GJR and APARCH models, to characterize and forecast financial time series volatility in Pakistan. The comparison is carried out by comparing symmetric and asymmetric GARCH models with normal and fat-tailed distributions for the innovations, over short and long forecast horizons. The forecasts are evaluated according to a set of statistical loss functions. Daily data on the Karachi Stock Exchange (KSE) 100 index are analyzed. The empirical results demonstrate that the use of asymmetry in the GARCH models and the assumption of fat-tail distributions for the innovations improve the volatility forecasts. Overall, EGARCH fits the best while the GJR model, with both normal and non-normal innovations, seems to provide superior forecasting ability over short and long horizons.

Keywords: APARCH; EGARCH; Fat-tailed distribution; Forecast; Forecast horizon; GARCH; GJR; KSE 100; Volatility.

\section{Introduction}

Financial markets play a crucial role in any country's economy. Monetary policies are generally based on stock exchange indices, foreign exchange rates, price indices, inflation rates, interest rates, etc. Further it is generally assumed that the ultimate goal for monetary policy is price stability. Empirical studies have concluded that a large change in prices today tends to be followed by a larger change in the financial sector for which a time series study needs to be conducted. One has to carry a time

\footnotetext{
* Dean of Sciences and Agricultures/Chairman, Department of Statistics, Bahauddin Zakariya University, Multan.

${ }^{* *}$ HEC Ph.D. Scholar, Department of Statistics, Bahauddin Zakariya University, Multan. *** Assistant Professor, Department of Statistics, Bahauddin Zakariya University, Multan.
} 
series study of all such financial changes. Some well-known characteristics are common to many financial time series. Even a cursory look at data suggests that some time periods are riskier than others resulting in a variation in the expected values of the error terms. Moreover, these risky times are not scattered randomly across quarterly or annual data. Instead, there is a degree of autocorrelation in the riskiness of financial returns. Volatility clustering is often observed. Financial time series often exhibit leptokurtosis, meaning that the distribution of their returns is fat-tailed. Moreover, the so-called leverage effect refers to the fact that changes in stock prices tend to be negatively correlated with changes in volatility. The econometric challenge is to specify how the information is used to estimate and forecast the mean and variance of the return, conditional on the past information. Currently the most powerful known techniques used to estimate and predict the volatility on high frequency data belong to a family of generalized conditional autoregressive heteroskedastic (GARCH) models. The goal of such models is to provide a volatility measure like a standard deviation that can be used in financial decisions concerning risk analysis, portfolio selection and derivative pricing.

Primarily, time varying heteroskedasticity is modeled by Engle (1982). He proposed the autoregressive conditional heteroskedastic (ARCH) process that allows the conditional variance to change over time as a function of past errors leaving the unconditional variance constant. Bollerslev (1986) extended his work and introduced the generalized autoregressive conditional heteroskedastic (GARCH) process. These models have been proved useful for modeling a variety of time series phenomena. However, both the models only control for the conditional heteroskedasticity, but they do not capture the so-called leverage effect. This led to the extension of nonlinear GARCH models e.g., the exponential GARCH (EGARCH) by Nelson (1991), GJR by Glosten, Jagannathan and Runkle (1993), the asymmetric power ARCH (APARCH) by Ding, Granger and Engle (1993), the Threshold GARCH of Zakoian (1994), the Quadratic GARCH (QARCH) by Santana (1995), etc. Although asymmetric models successfully capture the leverage effect, under the assumption of normal distribution of the innovation, they fail to capture the thick tail properties of financial time series. This has naturally led to the use of non-normal distributions, such as student- $t$, generalized error, normal Poisson, normallognormal, Bernoulli-normal, and skewed student- $t$ distributions (see Peters, 2001 and the references therein).

The forecasting performance of GARCH models has been assessed many times e.g., Pagan and Schwert (1990), Brailsford and Faff (1996) and Loudon, Watt and Yadav (2000). On the other hand, comparing normal 
densities with non-normal ones, has also been studied in several times e.g., see Hsieh (1989), Baillie and Bollerslev (1989), Peters (2001) and Lambert and Laurent (2001).

The main goal of present study is to evaluate the performance of different GARCH models in terms of their ability to characterize and predict out-of-sample volatility of financial time series in Pakistan. For this purpose, we compare the forecasting ability of GARCH, EGARCH, GJR and APARCH models with normal, student- $t$ and generalized error distribution (GED) innovations. The forecasting performance of such models is assessed through statistical loss functions. The estimates and forecasts are made on the KSE 100 index, because Pakistan's KSE 100 index is the best-performing stock market index in the world.

The plan of the paper is as follows: Section 2 discusses the models used in the study. Section 3 briefly describes the densities. In Section 4 we discuss forecast evaluation methods in terms of the statistical loss function to assess the forecast ability. All the empirical results and discussions are presented in Section 5 and some concluding remarks are made in Section 6.

\section{Volatility Models}

\subsection{The GARCH Process}

Let $y_{t}$ denote the price index at time $t=1,2, \ldots, T$ and $r_{t}=\ln \left(y_{t} / y_{t-1}\right) \times 100$ denote the rate of return from time $t$ to $t$ - 1 . Let $\varepsilon_{t}$ be a real valued discrete - process and $\Psi_{t}$ the information set ( $\sigma$-field) of all information through time $t$. The $\operatorname{ARMA}(k, l)$-GARCH $(p, q)$ process is then defined as in (1)-(2)

$$
\begin{aligned}
& r_{t}=\phi_{0}+\sum_{i=1}^{k} \phi_{t-i} r_{t-i}+\sum_{j=1}^{l} \theta_{t-j} \varepsilon_{t-j}+\varepsilon_{t}, \\
& \varepsilon_{t} / \Psi_{t-1} \sim N\left(0, \sigma_{t}^{2}\right), \\
& \sigma_{t}^{2}=\gamma_{0}+\sum_{i=1}^{q} \gamma_{i} \varepsilon_{t-i}^{2}+\sum_{j=1}^{p} \omega_{j} \sigma_{t-j}^{2},
\end{aligned}
$$

where $p \geq 0, q>0, \quad \gamma_{0}>0, \quad \gamma_{i} \geq 0$ for all $i=1,2, \ldots, q$ and $\omega_{j} \geq 0$ for $j=0,1, \ldots, p$. If $p=0$ the $\operatorname{GARCH}(p, q)$ process reduces to the 
$\mathrm{ARCH}(q)$ process and the conditional variance is simply a linear function of the past squared innovations only. If $p=q=0$ then the GARCH process is simply white noise with constant unconditional variance. The GARCH process defined in (1) is stationary

$$
\text { iff } \sum_{i=1}^{q} \gamma_{i}+\sum_{j=1}^{p} \omega_{j}<1
$$

Under the GARCH $(p, q)$ process, the one-step-ahead volatility forecast may be given as

$$
\hat{\sigma}_{T+1 / T}^{2}=\hat{\gamma}_{0}+\sum_{i=1}^{q} \hat{\gamma}_{i} \varepsilon_{T+1-i}^{2}+\sum_{j=1}^{p} \hat{\omega}_{j} \hat{\sigma}_{T+1-i}^{2}
$$

\subsection{EGARCH ModeI}

The exponential GARCH or EGARCH model involves the first introduction of an asymmetric effect on negative and positive shocks in an econometric model of volatility, by Nelson (1991). The specification for such a model is given as

$$
\ln \sigma_{t}^{2}=\gamma_{0}+\sum_{i=1}^{q}\left(\gamma_{i}\left|\eta_{t-i}-E\left(\eta_{t-i}\right)\right|+\beta_{i} \eta_{t-i}\right)+\sum_{j=1}^{p} \omega_{j} \sigma_{t-j}^{2}
$$

where $\eta_{t}=\frac{\varepsilon_{t}}{\sigma_{t}}$ is the standardized normal residual series.

The formulation in logarithm shares the usual positivity constraints on the parameters and also implies that the leverage effect is exponential rather than quadratic. The asymmetric effect is introduced by the non-linear function $\gamma_{i}\left|\eta_{t-i}-E\left(\eta_{t-i}\right)\right|+\beta_{i}\left(\eta_{t-i}\right)$ which is the function of both the magnitude and the sign of $\eta_{t}$. This specification has another advantage as compared to other asymmetric GARCH models; that is, it does not require any stationary constraints.

One step-ahead conditional variance forecast may be given as

$$
\hat{\sigma}_{T+1 \mid T}^{2}=\exp \left(\hat{\gamma}_{0}+\sum_{i=1}^{q}\left(\hat{\gamma}_{i}\left|\eta_{T+1-i}-E\left(\eta_{T+1-i}\right)\right|+\hat{\beta}_{i} \eta_{T+1-i}\right)+\sum_{j=1}^{p} \hat{\omega}_{j} \hat{\sigma}_{T_{T+1-i}}^{2}\right)
$$




\subsection{GJR Model}

Gloston, Jagannathan and Runkle (1993) also consider the impact of good and bad news by introducing indicator function in the symmetric GARCH model

$$
\sigma_{t}^{2}=\gamma_{0}+\sum_{i=1}^{q}\left(\gamma_{i} \varepsilon_{t-i}^{2}+\beta_{i} d_{t-i} \varepsilon_{t-i}^{2}\right)+\sum_{j=1}^{p} \omega_{j} \sigma_{t-j}^{2}
$$

where $d_{t}$ is the dummy variable and takes the value 0 when $\varepsilon_{t}$ is positive and 1 when $\varepsilon_{t}$ is negative. In other words the impact of $\varepsilon_{t}^{2}$ on the conditional variance is different when $\varepsilon_{t}$ is positive or negative.

The one-step-ahead volatility forecast for the GJR model may be given as

$$
\hat{\sigma}_{T+1 \mid T}^{2}=\hat{\gamma}_{0}+\sum_{i=1}^{q}\left(\hat{\gamma}_{i} \varepsilon_{T+1-i}^{2}+\hat{\beta}_{i} d_{T+1-i} \varepsilon_{T+1-i}^{2}\right)+\sum_{j=1}^{p} \hat{\omega}_{j} \hat{\sigma}_{T+1-j}^{2}
$$

\subsection{APARCH Model}

The GARCH $(p, q)$ model has been extended in various ways. Among the most interesting developments are the asymmetric power GARCH and $\operatorname{APARCH}(p, q)$ model (Ding, Granger and Engle, 1993), which allows to take account of both asymmetry and (possible) long memory property. The APARCH model can be expressed as

$$
\sigma_{t}^{\delta}=\gamma_{0}+\sum_{i=1}^{q} \gamma_{i}\left(\left|\eta_{t}\right|-\beta_{i} \eta_{t}\right)^{\delta}+\sum_{j=1}^{p} \omega_{j} \sigma_{t-j}^{\delta}
$$

where $p \geq 0, q>0, \gamma_{0}>0, \gamma_{i} \geq 0, \quad-1<\beta_{i}<1$ for all $i=1,2, \ldots, q$, $\omega_{j} \geq 0$ for all $j=1,2, \ldots, p \quad \delta>0$.

The covariance stationary condition for the model is

$$
\sum_{i=1}^{q} \gamma_{i}\left(\left|\eta_{t}\right|-\beta_{i} \eta_{t}\right)^{\delta}+\sum_{j=1}^{p} \omega_{j}<1
$$


Ding, Granger and Engle (1993) found that the closer $\delta$ is to 1, the larger is the memory of the process. Equivalently, this model couples the flexibility of a varying exponent with an asymmetry coefficient. Moreover, the APARCH model includes seven other ARCH extensions as special cases (see, Peter, 2001, for more details).

One step-ahead volatility forecast may be given as

$$
\hat{\sigma}_{T+1 \mid T}^{\delta}=\hat{\gamma}_{0}+\sum_{i=1}^{q} \hat{\gamma}_{i}\left(\left|\varepsilon_{T+1-i}\right|-\hat{\beta}_{i} \varepsilon_{T+1-i}\right)^{\delta}+\sum_{j=1}^{p} \hat{\omega}_{j} \hat{\sigma}_{T+1-j}^{\delta} \cdot
$$

\section{Densities}

A normal density for innovation was assumed in the ARCH process introduced by Engle (1982) and Bollerslev (1986) who extended the ARCH process into GARCH. Although the normal distribution is widespread, it cannot effectively describe the thick tails of stock returns, due to excess kurtosis. Bollerslev and Wooldridge (1992) proposed quasi-maximum likelihood (QML) procedure which is robust to departures from normality. Although the QML estimator is consistent, it is inefficient for non-normality distributed data as the degree of inefficiency increases with the degree of departure from normality (Engle and Gonzalez-Rivera, 1991). This leads to the use of other distribution functions, such as the student- $t$ by Bollerslev (1987) and generalized error distribution (GED) by Nelson (1991) to mode1 tail thickness by a parameter, called degree of freedom.

\subsection{Standardized Student-t Distribution}

Bollerslev (1987) proposed the standardized student- $t$ distribution with $v>2$ degrees of freedom,

$$
f\left(\eta_{t}\right)=\frac{\Gamma((v+1) / 2)}{\Gamma(v / 2) \sqrt{\pi(v-2)}}\left(1+\frac{\eta_{t}^{2}}{v-2}\right)^{-\frac{v+1}{2}},
$$

where $\Gamma$ (.) is the gamma function. The degree of freedom represents the parameter to be estimated. The $t$-distribution is symmetric around zero and for $v>4$ the conditional kurtosis equals $3(v-2)(v-4)^{-1}$, which exceeds the normal value of 3 , but for $v \rightarrow \infty$ the density of standardized student- $t$ distribution converges to the density function of the standardized normal distribution. 


\subsection{Generalized Error Distribution} (GED)

Nelson (1991) suggested the use of the generalized error distribution

$$
f\left(\eta_{t}\right)=\frac{v \exp \left(-0.5\left|\eta_{t} / \lambda\right|^{v}\right)}{2^{\left(1+\frac{1}{v}\right)} \Gamma\left(v^{-1}\right) \lambda} \quad v>0
$$

where $v$ is the tail-thickness parameter and $\lambda \equiv\left[2^{(-2 / v)} \Gamma(1 / v) / \Gamma(3 / v)\right]^{1 / 2}$. When $v=2, \quad \eta_{t}$ is standard normally distributed. For $v<2$, the distribution of $\eta_{t}$ has thicker tails than the normal distribution (e.g., for $v=1, \eta_{t}$ has double exponential distribution) while for $v>2$ the distribution of $\eta_{t}$ has thinner tails than the normal distribution (e.g., for $v=\infty, \eta_{t}$ has a uniform distribution on the interval $(-\sqrt{3}, \sqrt{3})$ (see Nelson, 1991). The conditional kurtosis is given by $(\Gamma(1 / v) \Gamma(5 / v)) /(\Gamma(1 / v))^{2}$.

Notice that the choice of a density has a particular impact on some models, for example in EGARCH the value of $E\left|\eta_{t}\right|$ depends on the density function for the standard normal distribution

$$
E\left(\eta_{t-i}\right)=\sqrt{\frac{2}{\pi}}
$$

for student- $t$ distribution

$$
E\left(\left|\eta_{t-i}\right|\right)=\frac{2 \Gamma\left(\frac{1+v}{2}\right)^{2} \sqrt{(v-2)}}{1+\sqrt{\pi}(v-1) \Gamma(v / 2)},
$$

for GED

$$
E\left(\left|\eta_{t-i}\right|\right)=\lambda 2^{1 / v} \frac{\Gamma(2 / v)}{\Gamma(1 / v)}
$$




\section{Forecast Evaluation Methods}

The comparison of forecasting performance of GARCH models requires the actual volatility denoted by $\sigma_{t}^{2}$. As such, it provides the natural benchmark for forecast evaluation purposes. A common model-free indicator of volatility is the daily squared return. However, one can obtain a more accurate measure by following an idea proposed by Merton (1980) and Schwert (1989) and formalized by Andersen and Bollerslev (1998). They argued that the single squared change is a noisy indicator for the latent volatility in the period, because the idiosyncratic component of a single change is large. The noise is reduced by taking the sum of all squared intraperiod changes, and the smaller the sub-period, the larger the noise reduction. Since the highest frequency available to us is daily data, this idea results in the use of the daily squared return $\sigma_{t}^{2}=r_{t}^{2}$ as actual volatility.

We have summed the daily realized volatility over the $k$-days to obtain the volatility at $k$-step-ahead (for $k>1$ ) i.e. $\sigma_{T+k}^{2}=\sum_{j=1}^{k} \sigma_{T+j}^{2}$. Similarly, $k$-step-ahead volatility forecast $\hat{\sigma}_{T+k \mid T}^{2}$ is the aggregated sum of the forecasts made at time Ti.e. $\hat{\sigma}_{T+k \mid T}^{2}=\sum_{j=1}^{k} \hat{\sigma}_{T+j \mid T}^{2}$.

The evaluation of forecast ability of competing volatility models is not an easy task, as pointed by Bollerslev, Engle and Nelson (1994), and Lopez (2001), and there does not exist an exceptional measure of selecting the best model. Hansen, Lunde and Nason (2003b) applied the Model Confidence Set (MCS) procedure of Hansen, Lunde and Nason (2003a) to a set of volatility models in order to pick the 'best' forecasting model, amongst case volatility models. As in this approach, the performance of a forecast may be evaluated by using an out-of-sample evaluation under a loss function specified by the user. But like many researchers (e.g., Peter, 2001 and Marcucci, 2005), this paper simply uses different statistical loss functions, available in literature for volatility forecast evaluation. These loss functions will be used as diagnostic tools on the forecasting model.

To assess the forecast ability of different models, the paper also uses some statistical loss functions that have different interpretations. These are given as: 
1. $\quad$ MSE $1=\frac{1}{b+1} \sum_{t=T}^{T+h}\left(\sigma_{t+1}-\hat{\sigma}_{t+1 \mid T}\right)^{2}$

2. $\quad M S E 2=\frac{1}{b+1} \sum_{t=T}^{T+h}\left(\sigma_{t+1}^{2}-\hat{\sigma}_{t+1 \mid T}^{2}\right)^{2}$.

3. $\quad M A E 1=\frac{1}{b+1} \sum_{t=T}^{T+h}\left(\left|\sigma_{t+1}^{2}-\hat{\sigma}_{t+1 \mid T}^{2}\right|\right)$.

4. $\quad M A E 2=\frac{1}{b+1} \sum_{t=T}^{T+h}\left(\left|\sigma_{t+1}-\hat{\sigma}_{t+1 \mid T}\right|\right)$.

5. $\quad M A P E=\frac{1}{b+1} \sum_{t=T}^{T+h}\left(\left|\frac{\sigma_{t+1}^{2}-\hat{\sigma}_{t+1 \mid T}^{2}}{\sigma_{t+1}^{2}}\right|\right)$.

6. TIC $=\frac{\sqrt{\frac{1}{h+1} \sum_{t=T}^{T+h}\left(\sigma_{t+1}^{2}-\hat{\sigma}_{t+1 \mid T}^{2}\right)^{2}}}{\sqrt{\frac{1}{b+1} \sum_{t=T}^{T+b}\left(\sigma_{t+1}^{2}\right)}+\sqrt{\frac{1}{b+1} \sum_{t=T}^{T+h}\left(\hat{\sigma}_{t+1 \mid T}^{2}\right)}}$.

7. Mincer-Zarnowitz $R^{2}$.

8. $\quad R^{2} L O G=\frac{1}{b+1} \sum_{t=T}^{T+h}\left(\log \left(\sigma_{t+1}^{2} / \hat{\sigma}_{t+1 \mid T}^{2}\right)\right)^{2}$.

9. $\quad H M S E=\frac{1}{b+1} \sum_{t=T}^{T+h}\left(\sigma_{t+1}^{2} / \hat{\sigma}_{t+1 \mid T}^{2}-1\right)^{2}$

In the above cases $b$ is the forecast horizon.

The first two measures are the mean square error (MSE). These forecast error statistics depend on the scale of the dependent variable. The criteria (3), (4) and (5) are the mean absolute error (MAE) and mean absolute percentage errors (MAPE), respectively. The MSE's are more sensitive to outliers than MAE's. The measure in (6) is the Theil inequality 
coefficient (TIC) which is scale invariant. It always lies between zero and one, where zero indicates a perfect fit. The loss function in (7) is computed in Mincer-Zarnowitz regressions (Mincer-Zarnowitz 1969), by regressing the actual variance $\sigma_{T+k}^{2}$ on the constant and forecasted variance $\hat{\sigma}_{T+k \mid T}^{2}$,

$$
\sigma_{T+k}^{2}=a+b \hat{\sigma}_{T+k \mid T}^{2}+v_{T+k}
$$

The statistic $\mathrm{R}^{2}$ from this regression provides the proportion of variance explained by the forecast i.e. the higher the $R^{2}$, better the forecasts. The $\mathrm{R}^{2} \mathrm{LOG}$, named by Pagan and Schwert (1990) as the logarithmic loss function, penalizes volatility forecasts asymmetrically in low and high volatility periods. The loss function in (9) is the k-adjusted MSE (HMSE), proposed by Bollerslev and Ghysels (1996).

\section{Empirical Results and Discussions}

\subsection{Data and Methodology}

In this section, we describe the data and our methodology. The whole sample consists of the KSE 100 index of Pakistan closing prices from January 1, 2002 to August 31, 2006, for a total of 1218 observations. The estimation process is run using four years of data (2002-2005) while the remaining eight months (January 1, 2006 to August 31, 2006) data are used for the evaluation of the out of sample forecast performance. The indices prices are transformed into their rates of returns.

First of all, the statistical properties of returns are assessed through means of coefficients of skewness and kurtosis, Jarque-Bera test of normality, ARCH LM test and Ljung-Box test on the squared residuals to check the presence of typical stylized facts.

Table-5.1: Descriptive Statistics of $r_{t}$

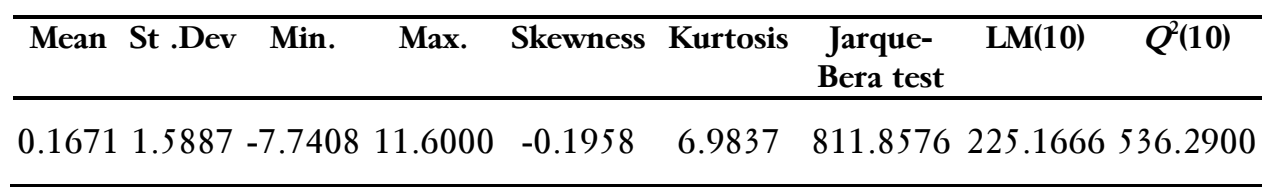

Table-5.1, represents the descriptive statistics of $r_{t}$. The Jarque-Bera statistic is high due to excess kurtosis and negative skewness, indicating the 
non-normality of the distribution. Moreover, LM (10) statistics is the ARCHLM test proposed by Engle (1982), $Q^{2}(10)$ is the Ljung-Box test statistics on the squared residuals up to lag of 10 . Under the null of no serial correlation, the high values for both the statistics indicate the presence of $\mathrm{ARCH}$ effect in the conditional variance.

For the identification of the mean model, we have followed the BoxJenkins methodology. A number of tentative models with increasing ARMA orders and increasing GARCH orders have been estimated. Appropriate models are identified using autocorrelation function (ACF), partial autocorrelation function (PACF) and Ljung-Box statistics of the standardized residuals and the squared standardized residuals and ARCH-LM test. Through this exercise, a GARCH $(1,1)$ process is found to be the best model for conditional variance. The final model amongst the models, satisfying the diagnostics is selected on the basis of Akiake information criterion (AIC) and Schwarz's Bayesian information criterion (BIC) given in the Appendix. The selected model is given as

$$
r_{t}=\phi_{0}+\phi_{9} r_{t-9}+\varepsilon_{t}
$$

Table-1, presents the estimation results for the parameters for the mean mode1, GARCH, GJR, EGARCH and APARCH models with three distributions: normal, student- $t$ and GED. Asymptotic k-consistent standard errors are given in parentheses. To estimate and forecast volatility, we use the popular software, EViews 5.0 


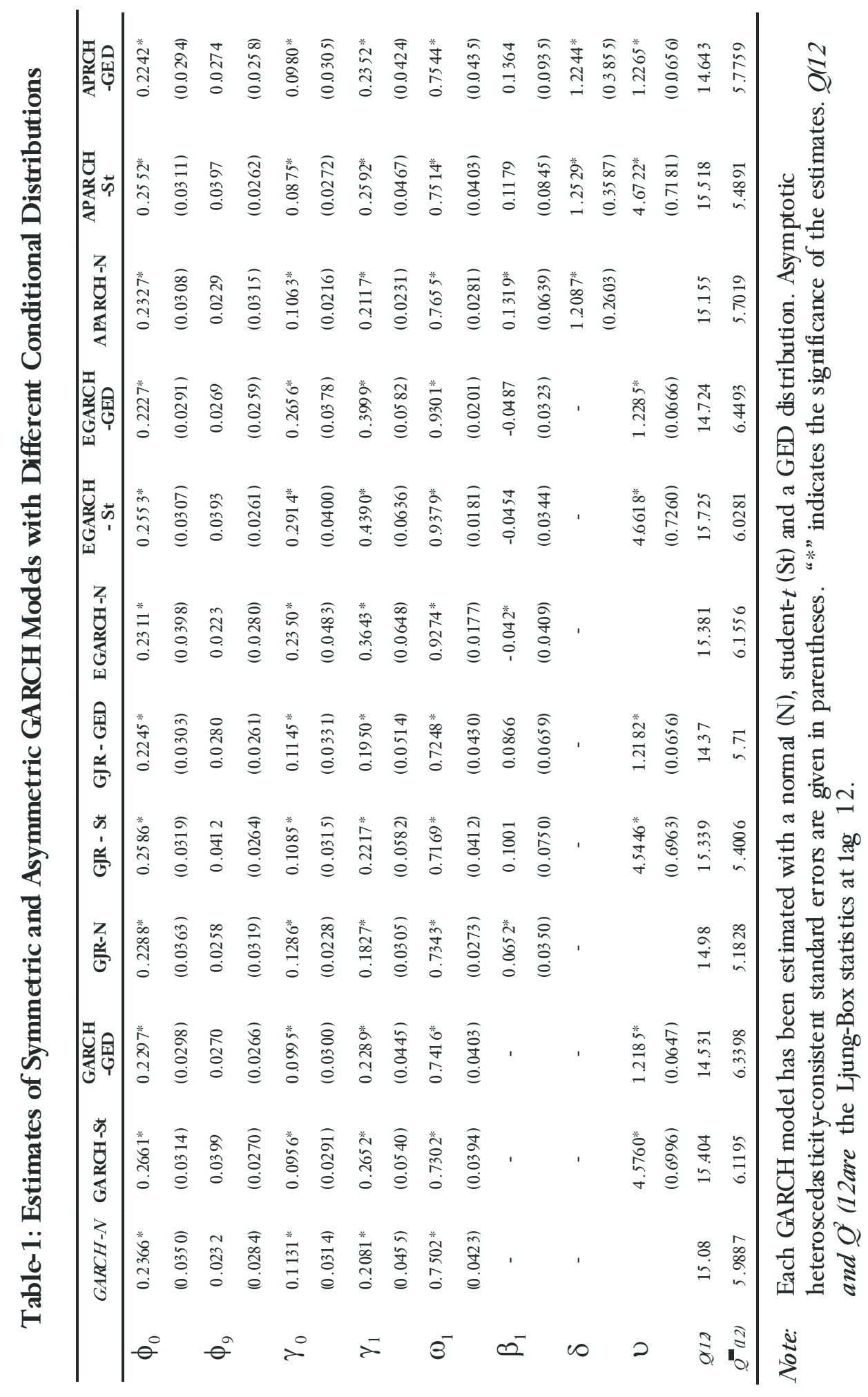


Regarding the conditional mean, $\hat{\phi}_{0}$ is highly significant for all the models. However, $\hat{\phi}_{9}$ is non-significant, although we do not drop this parameter because by doing so, the ACF of the standardized residuals becomes significant at lag 9. Moreover, as our main focus is on the forecasts of volatility and by dropping this parameter, the forecast's accuracy reduces. The conditional variance estimates show that all the parameters are highly significant except asymmetric parameters in the cases of student- $t$ and GED distributions. In addition, for the student- $t$ distribution, the values of shape parameter $v$ for GARCH, EGARCH, GJR and APARCH clearly indicate the typical fat-tail behavior of financial returns. Moreover, for the GED, the estimates clearly suggest that the conditional distribution has fatter tails than the normal distribution, since the shape parameters for GARCH, EGARCH, GJR and APARCH have values that significantly between 1 and 2 indicating the conditional distribution of KSE 100 index is indeed fat-tailed. Ljung Box statistics at $\operatorname{lag} 12, Q^{(12)}$ and $Q^{2}(12)$ on the standardized residuals and the squared standardized residuals respectively, are nonsignificant indicating that all these models adequately described the dynamics of the series. 


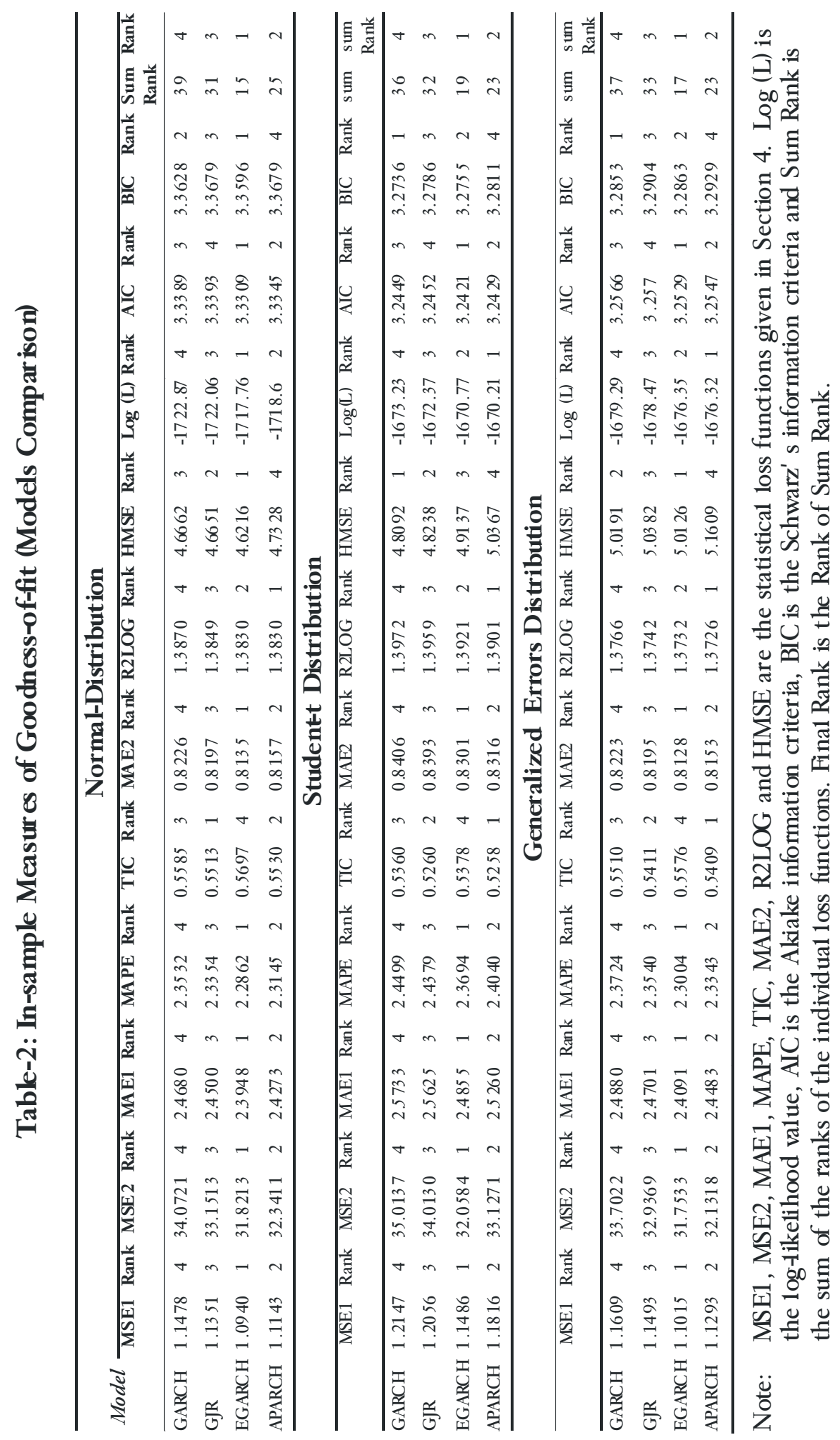


Table-2 shows the model comparison in terms of measures of goodness of fit. The results demonstrate that the performance of asymmetric GARCH models with all the three distributions justified the use of asymmetric GARCH models to estimate the series as highlighted by the values of the log-likelihood. According to AIC, EGARCH perform the best in all the three cases. According to the statistical loss functions considered in this study, the EGARCH model with normal and non-normal innovations fits the best, since the sum of the ranks is the smallest. The second best model is the APARCH. However, the performance of GARCH model with all the three distributions is poorest, as the sum of the ranks of all the measures is highest in each case. 


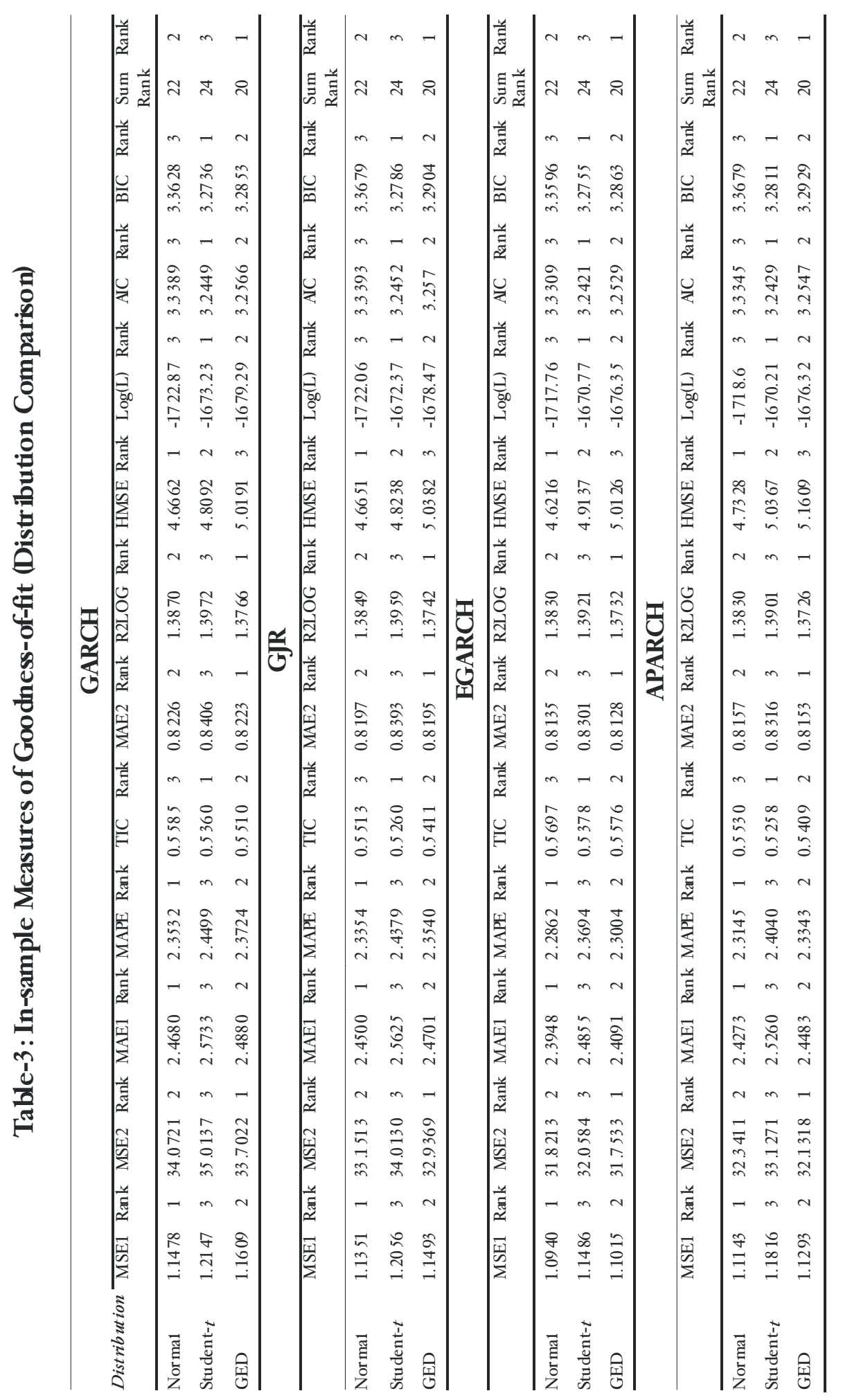


Table-3 shows the distribution comparison in terms of measures that calculate goodness of fit. The results show that the overall comparison is difficult. According to the log-likelihood, AIC and BIC, the competing models fit the best with fat-tailed distributions and prominent student- $t$, while the symmetric and asymmetric GARCH models with normal distribution perform the poorest. According to other measures, all the competing models with student- $t$ innovations perform the poorest. Overall, on the basis of all the measures, all the competing models fit best on the series with GED innovations. 


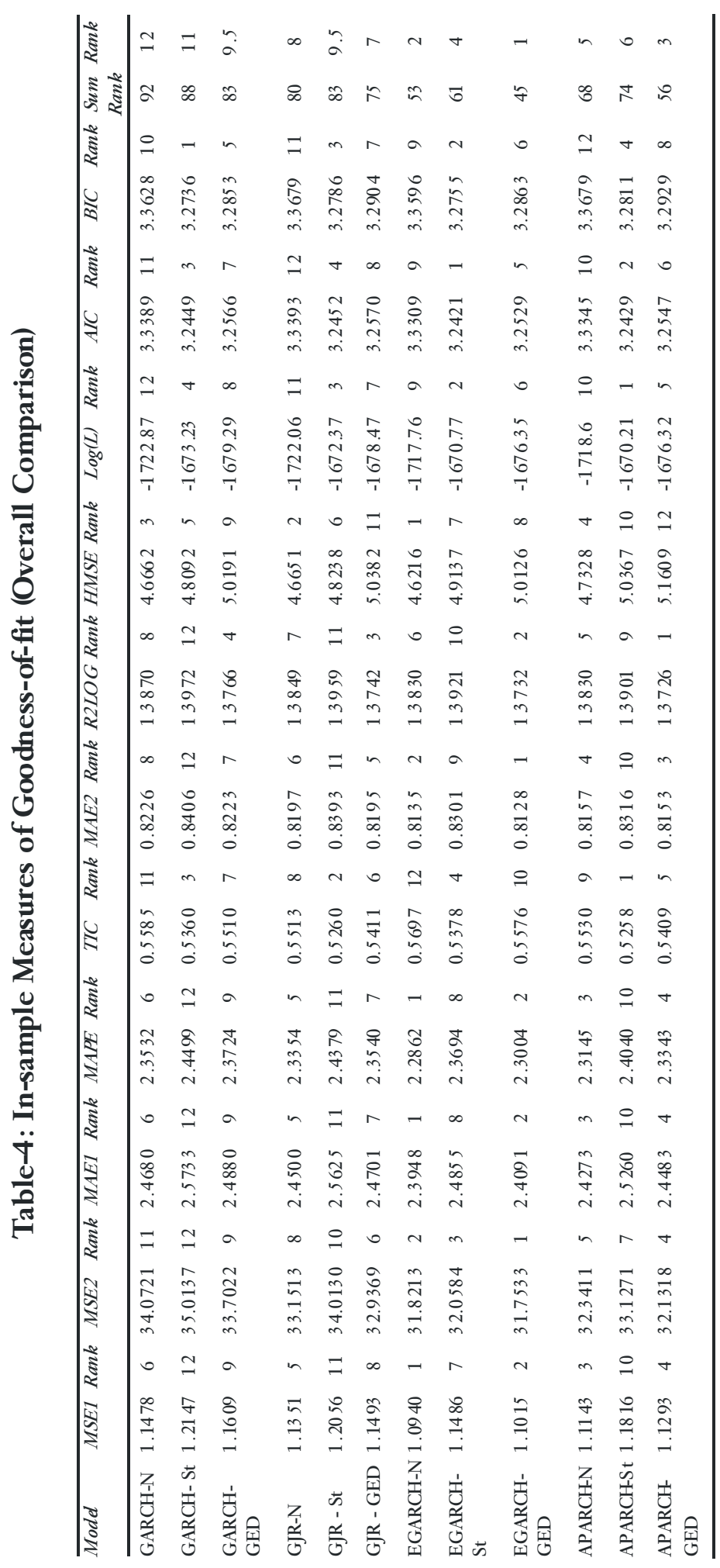


Table-4 shows the overall in-sample measures of goodness of fit. The overall comparison shows that the largest log-likelihood is given by the APARCH model with student- $t$ innovations, while AIC indicates that the best model is EGARCH with student- $t$ innovations. Overall, the sum of the ranks of all statistical loss functions show that the EGARCH models with GED and normal innovations respectively fit the best followed by the EGARCH and APARCH models while the performance of the GARCH model is the poorest.

\subsection{Forecast Evaluation}

The main goal of our study is to compare the forecasting ability of different GARCH models. Such a comparison has been carried out by comparing the volatility forecasts at one-, five- ten-, fifteen- and twentysteps-ahead. Forecasting ability of competing GARCH models is reported by ranking according to the statistical loss functions given in section 4 through Table-5 to Table-11. We have compared the results in terms of model comparisons and distribution comparisons at all the one-, five- ten-, fifteenand twenty-steps-ahead forecast horizons. But the scope of the present paper has been limited to the case of ten-steps, as the rest of the cases follow a similar pattern. However, the total comparison is given for all the forecast horizons. Finally the best performing model is selected by ranking the sum of the ranks of the individual loss functions. 


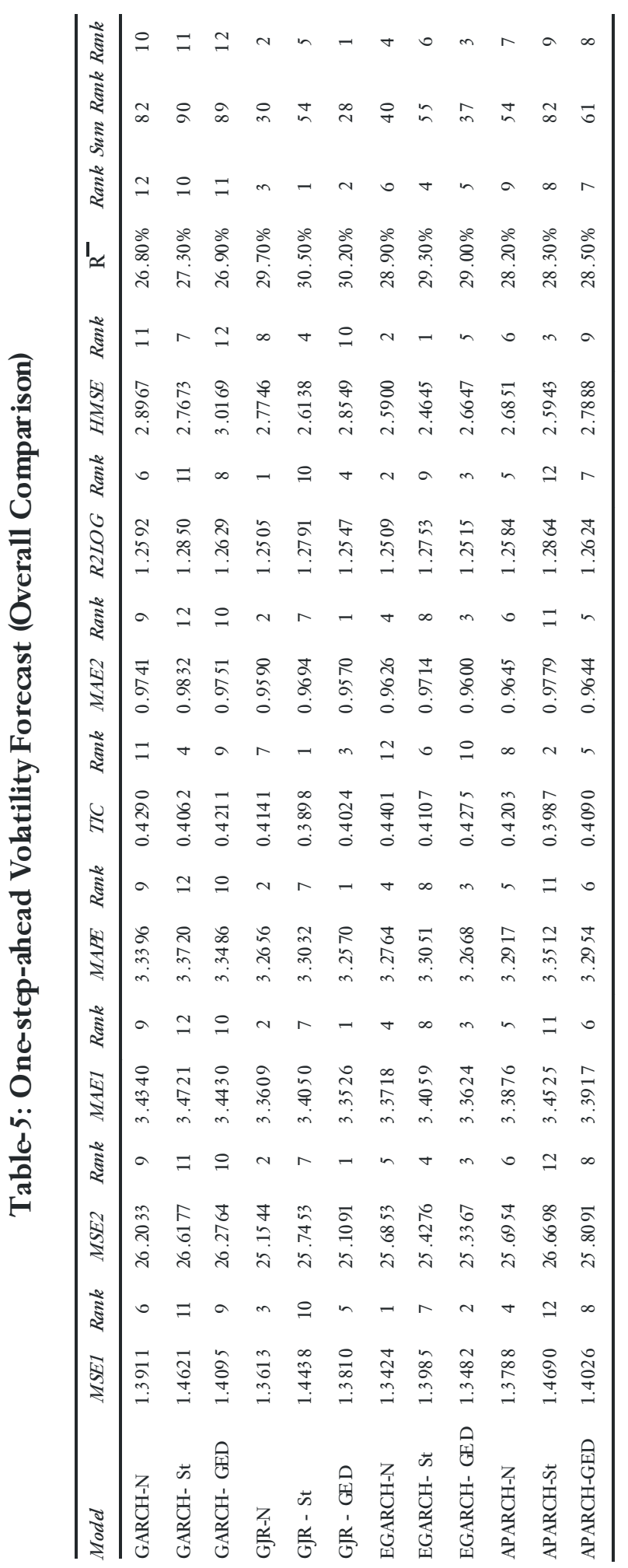




\subsubsection{One-step-ahead Forecast Evaluation}

Table- 5 shows the forecast evaluation at one step ahead. The mode1 comparison recommends that asymmetric GARCH models perform the best for all the three distributions made obvious by final ranks. For all the three distributions, the pattern of the ranks of the final ranks (the ranks of the sum of the individual loss functions) is $(4,1,2,3)$, for GARCH, GJR, EGARCH and APARCH respectively. This indicates that the first best model is the GJR and the second best model is EGARCH. APARCH provide less satisfactory results while symmetric GARCH, clearly, gives the poorest forecasts.

The comparison between densities is harder because results vary across models. The symmetric GARCH and APARCH show the pattern of the ranks of the final ranks as $(1,3,2)$, for normal, student- $t$ and GED respectively, indicating the best results are obtained with normal innovations. While GJR and EGARCH gives the final ranks as $(2,3,1)$ for normal, student- $t$ and GED respectively revealing the best results with GED innovations. At one-step-ahead, the forecasting ability of all the competing models with student- $t$ innovation is the poorest.

The overall comparison of the forecasting performance of the competing models shows that GJR model with GED innovations seems to perform the best. 


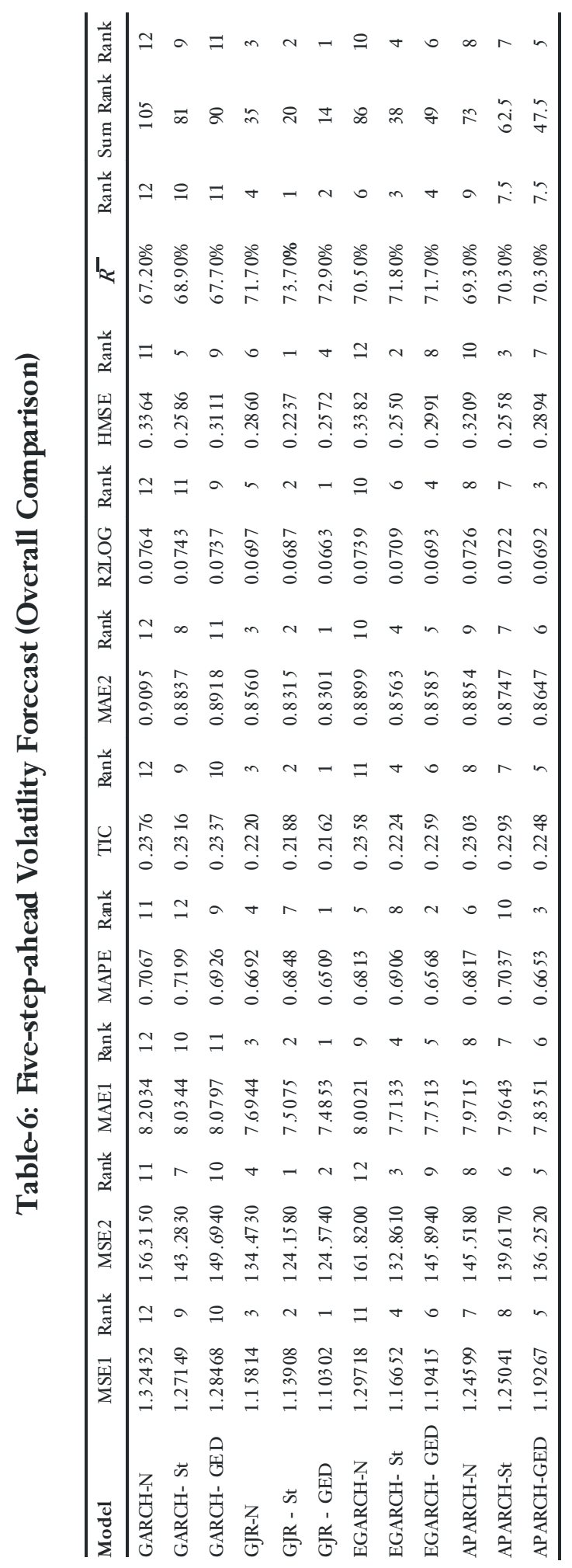




\subsubsection{Five- step-ahead Forecast Evaluation}

Table-6 shows the forecast comparison at five-steps-ahead. The model comparison gives a pattern similar to the final ranks at one-stepahead forecast horizon. So, the use of asymmetric GARCH model versus the symmetric GARCH is strongly recommended. For all three distributions the first best model is again the GJR and the second best model is EGARCH. APARCH provides less satisfactory results while symmetric GARCH clearly gives the poorest forecasts.

The comparison between densities led to the use of non-normal densities since all the competing models give better forecasts with fat-tail distributions. The symmetric GARCH and EGARCH show the pattern of the ranks of the final ranks as $(3,1,2)$, for normal, student- $t$ and GED respectively, indicating the best results lie with student- $t$ innovations. Moreover, GJR and APARCH give the final ranks as $(2,3,1)$ for normal, student- $t$ and GED respectively revealing the best results with GED innovations. At five-steps-ahead the forecasting ability of all the competing models with normal innovation is poorest.

Overall results illustrate that the GJR model with GED is again the most successful model to forecast the volatility of KSE 100 at five stepsahead. 


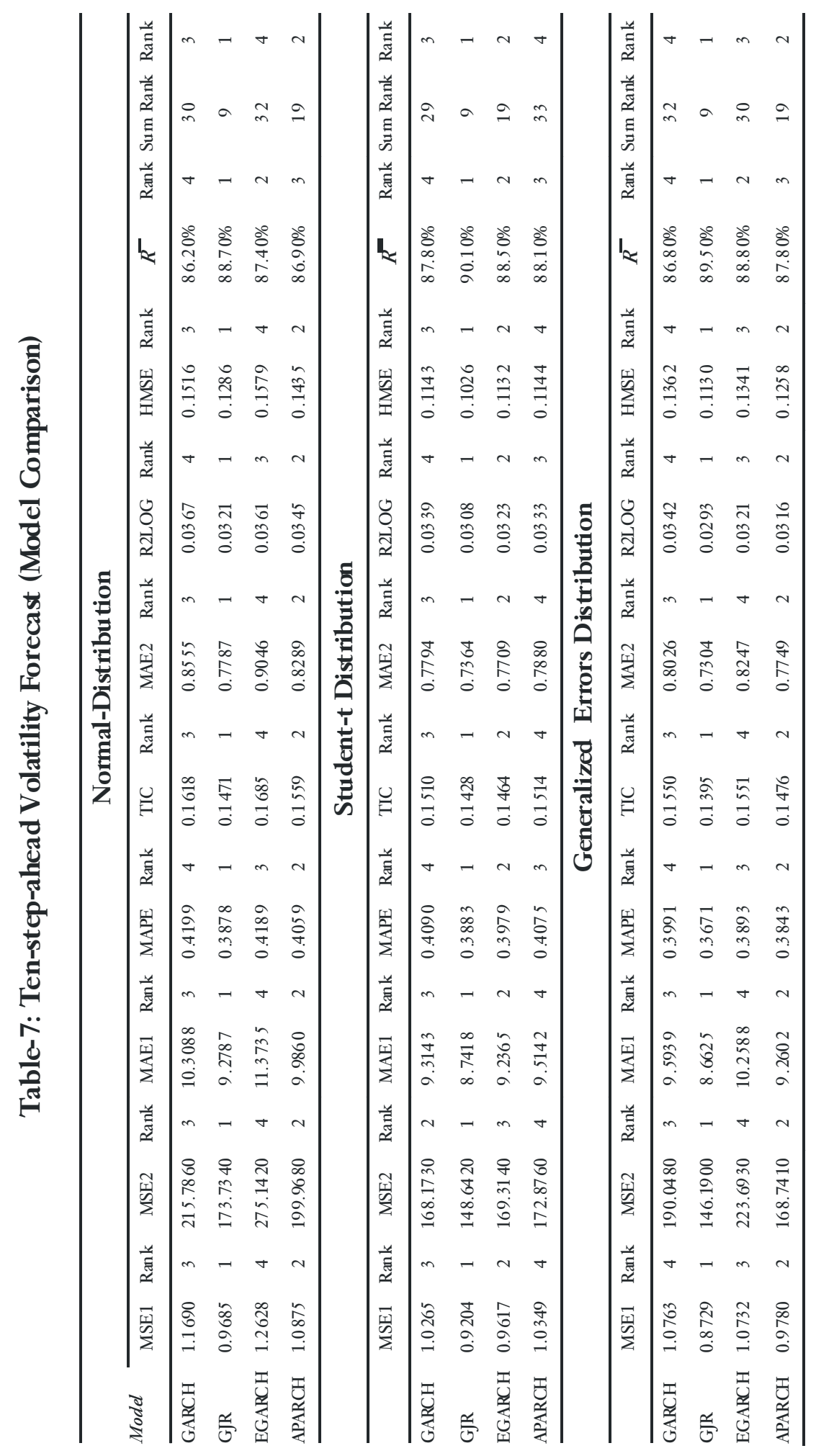




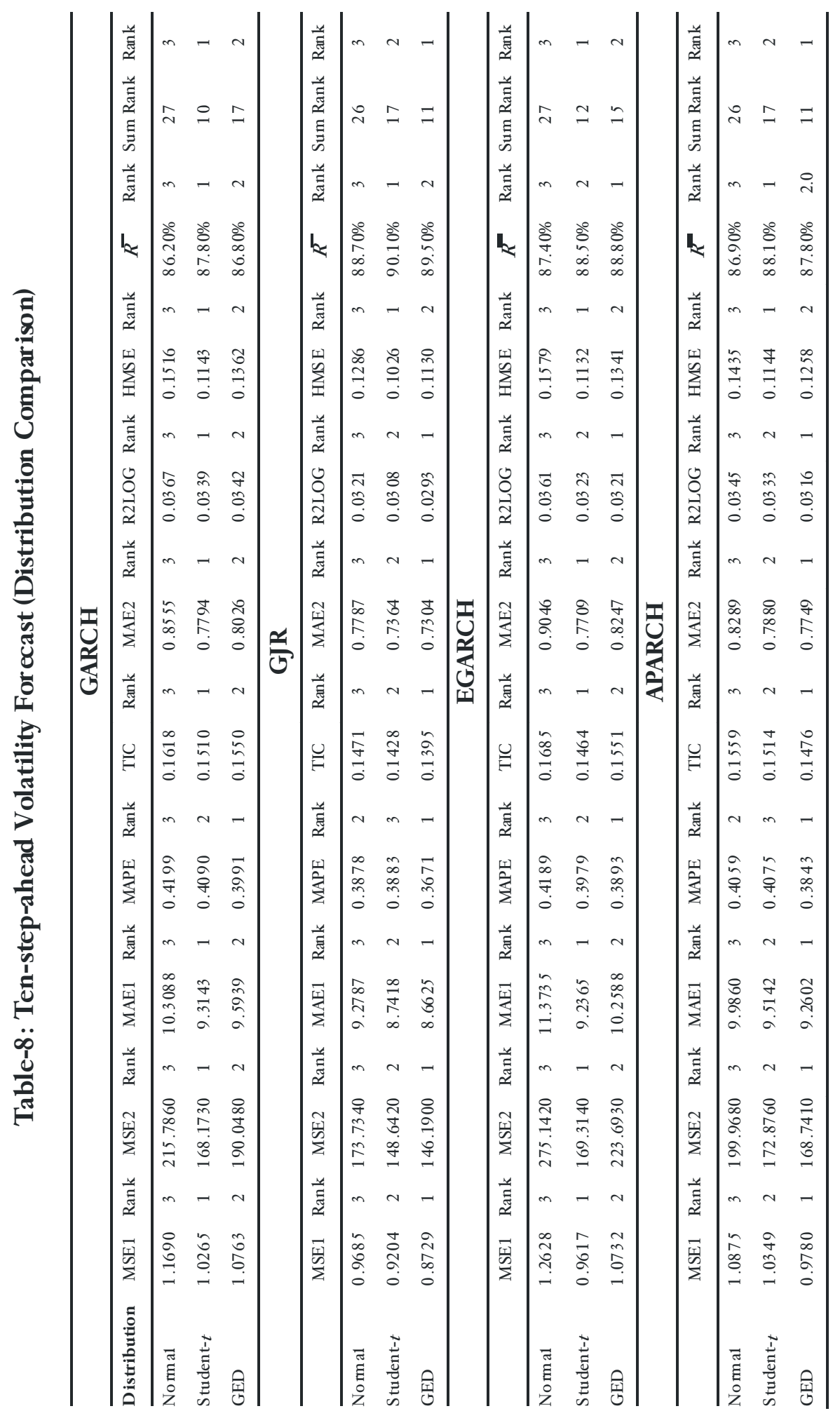




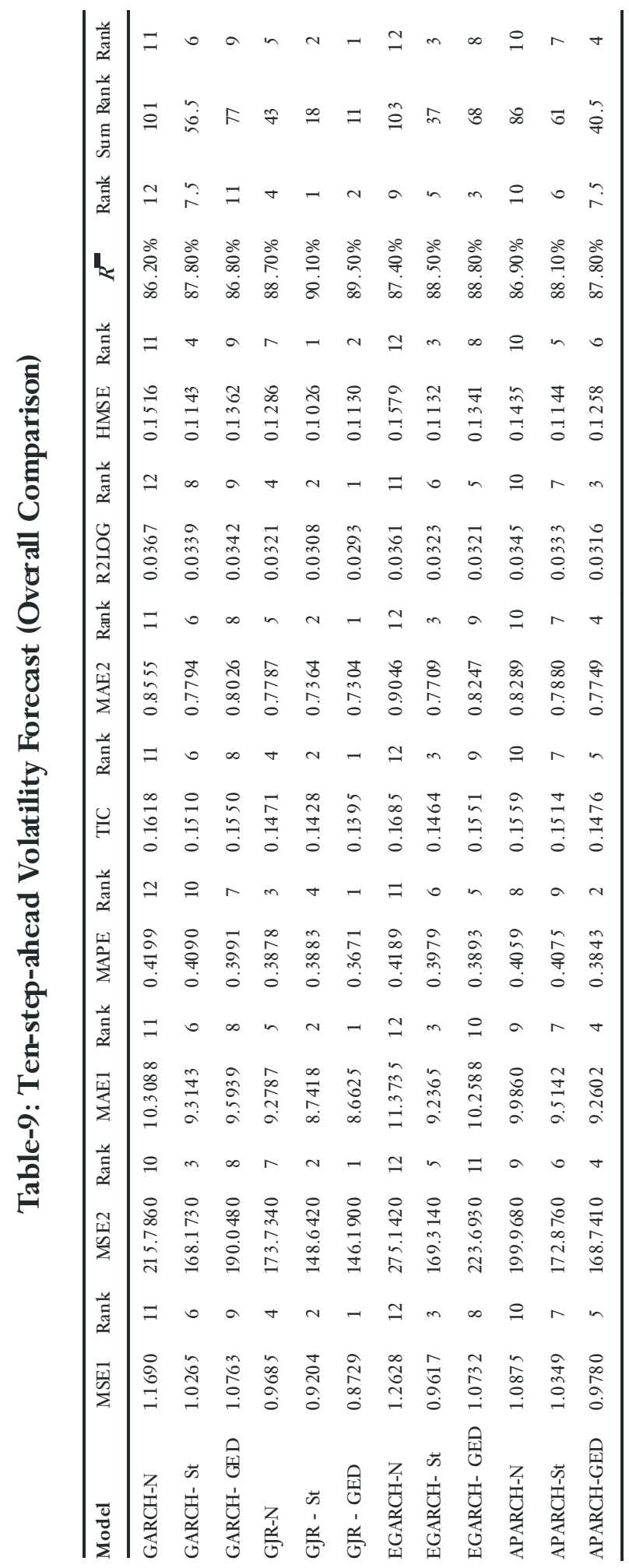




\subsubsection{Ten - step-ahead Forecast Evaluation}

The model comparison at the ten-step-ahead forecast horizon is given by Table-7. The model that reveals the best forecasting ability lies again with GJR for all the three distributions as highlighted by all the loss functions given in Table-7. The comparison between the other models is complicated because the results are conflicting. For the normal and GED, the second best model is APARCH while it performs the poorest with student- $t$. On the other hand the performance of EGARCH is better with student- $t$ versus normal and GED.

Table- 8 shows the distribution comparison. The results favor the use of non-normal densities, since all the symmetric and asymmetric GARCH models provide better forecasting performance with non-normal innovations. However, within non-normal distributions GARCH and EGARCH better perform with student- $t$ distribution while GJR and APARCH better perform with GED innovations.

Yet again, overall the preeminent model is GJR with GED innovations as obvious by Table-9. All statistical loss functions except HMSE and $R^{2}$ strongly support the use of GJR with GED innovations to forecast the volatility of KSE 100 at the ten-step-ahead forecast horizon. The second best model is also GJR with student- $t$ innovations. 


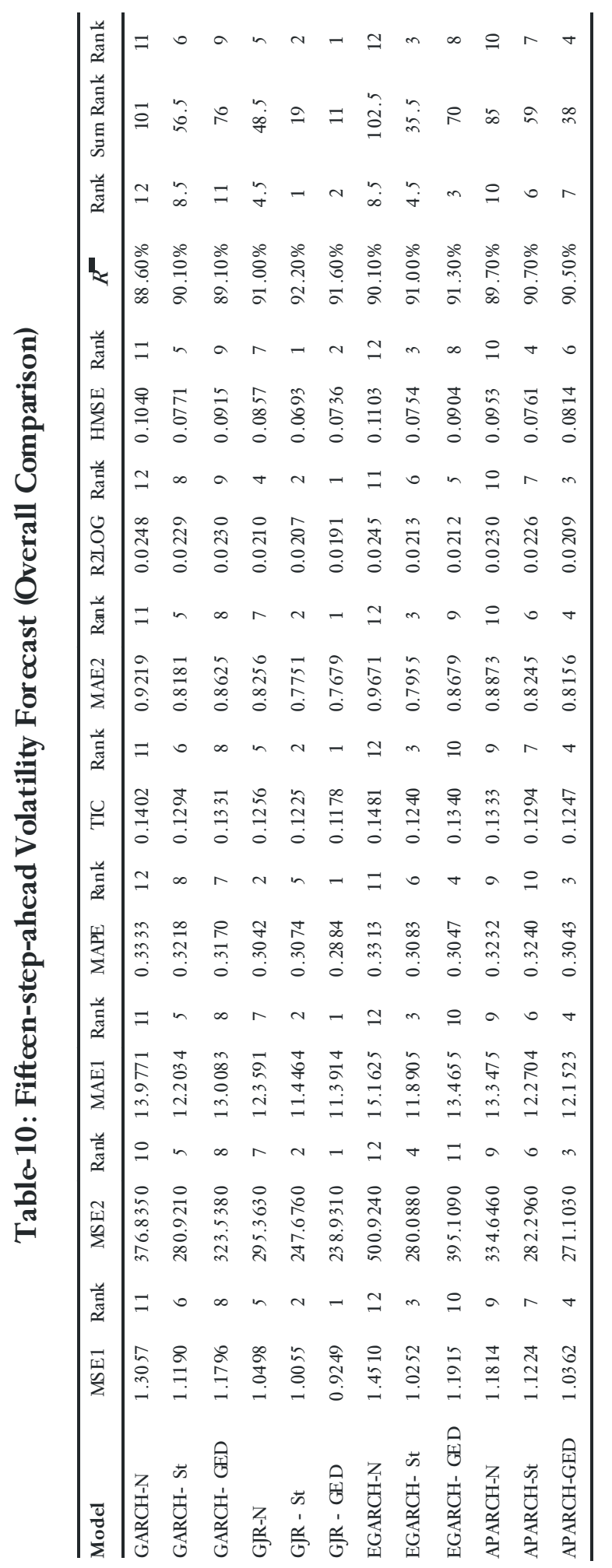




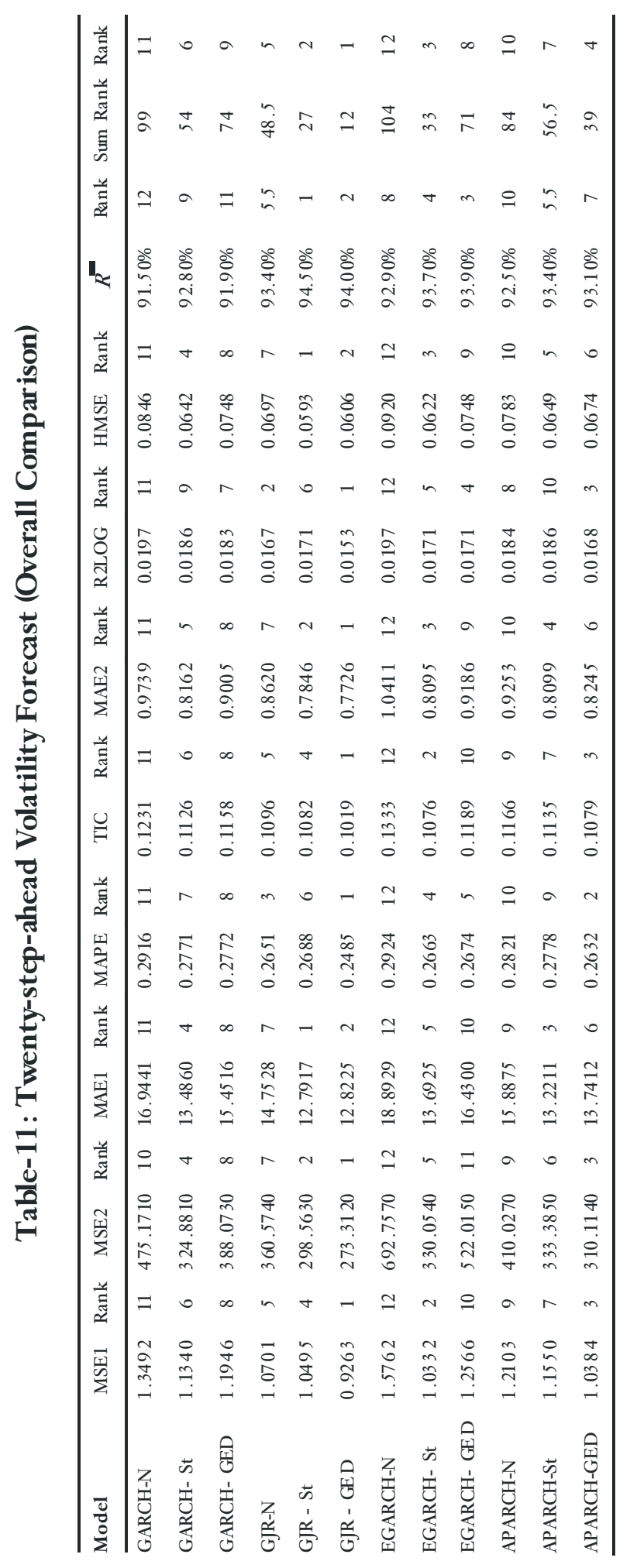




\subsubsection{Fifteen- step-ahead Forecast Evaluation}

The model comparison at fifteen-step-ahead volatility forecast also shows that GJR provides the best forecasting ability for all the three distributions.

The forecasting ability of all the symmetric GARCH and asymmetric GARCH models is better with non- normal densities than with normal densities.

The overall performance of GJR is the best in the model comparison and in the densities comparison.

\subsubsection{Twenty- step-ahead Forecast Evaluation}

At twenty-step-ahead forecasting, the competing models reveal the same forecasting performance with normal and non-normal densities as at the fifteen-step-ahead forecast horizon. So, similar conclusions may be drawn as at fifteen-step-ahead forecast horizon.

It is conspicuous that the $R^{2}$ is higher when using non-normal distributions and is highest when using a student- $t$ distribution at all the forecast horizons. Its value also increases from shorter to longer forecast horizons e.g., the highest value at one-day forecast horizon is $30.50 \%$ and is $94.50 \%$ at twenty-days forecast horizon. But it does not mean the forecast is inadequate at shorter forecast horizons, as explained by Anderson and Bollerslev (1998) and Klaassen (2002). The primary reason for the low $R^{2}$ at shorter forecast horizons is the noise in the observed volatility measure. As discussed in Section 4, this noise can be reduced by taking the sum of squared changes over sub-periods. To give an indication of the magnitude of the effect of this noise reduction on $R^{2}$, Anderson and Bollerslev compute the $R^{2}$ for a $\operatorname{GARCH}(1,1)$ model on daily mark/dollar and yen/dollar exchange rates using a single squared daily changes and using the sum of 288 squared five-minute changes in a day. The $R^{2}$ increases and they conclude that GARCH does provide good volatility forecasts despite the low $R^{2}$ that is typically obtained using the single squared change. For the purpose of this paper, the argument also explains why the $R^{2}$ is higher for the longer horizons than for the shorter horizons; in the return series the noise has been reduced in the twenty-day realized volatility by using twenty instead of one squared returns. Further the $R^{2}$ is also the highest for the GJR model with student- $t$ innovations, at all the one-day, five-day, ten-day, fifteen-day and twenty-day forecast horizons. 
These results generally recommend that volatility forecasts of the KSE 100 index may be improved by using asymmetric GARCH models with non-normal distributions at both short and long forecast horizons. It is also apparent that the GJR model with GED innovations outperforms the other models, at all the forecast horizons.

\section{Conclusion}

The essential goal of this paper was to compare the performance of several GARCH-type models (GARCH, EGARCH, GJR and APARCH) in estimating and forecasting the volatility of the KSE 100 index. Such a comparison is carried out by comparing one-day, five- day, ten-day, fifteenday and twenty-day-ahead volatility forecasts. In addition, all the models are estimated assuming both normal and fat-tailed distributions such as student$t$ and GED for the innovations. The comparison was focused on different aspects: the difference between symmetric and asymmetric GARCH (i.e. GARCH versus EGARCH, GJR and APARCH), and the difference between normal and fat-tailed distributions.

Our results show that traceable improvements can be made when an asymmetric GARCH model is used in estimating volatility of the KSE 100 return series. Generally, according to the statistical loss functions, among the competing models, EGARCH and APARCH fit the series better than GJR models. Also, the symmetric GARCH model provides the poorest results to fit the series. All the models with GED innovations fit the series the best. Overall, on the basis of rank of the sum of the ranks of individual loss functions, EGARCH with GED fits the best.

Overall, the empirical results show that GJR with all the three distributions seems to provide superior forecasting performance at all oneday, five day ten-day, fifteen-day and twenty-day-ahead volatility forecasts horizons according to the statistical loss functions. So, it may be concluded that the asymmetric effect is central to estimating the quadratic effect for forecasting. The symmetric GARCH model performs poorly according to the statistical loss functions, especially at shorter forecast horizons. Moreover, non-normal distributions, generally, provide better out-of-sample results than the normal distribution.

Further, according to the different statistical loss functions that evaluate out-of-sample forecasts, the GJR model with GED innovations seems to provide superior forecast ability at both shorter and longer forecast horizons. So, it may be concluded that it is the best way to forecast volatility of KSE 100 index is at shorter and longer forecast horizons. 
Appendix

Table-A: Model Selection

\begin{tabular}{cccc}
\hline Models & LogL & AIC & BIC \\
\hline ARMA(9, 0)-GARCH $(1,1)$ & -1722.870 & 3.3388 & 3.3627 \\
ARMA(2, 2)-GARCH $(1,1)$ & -1731.888 & 3.3395 & 3.3775 \\
ARMA(3, 0)-GARCH $(1,1)$ & -1749.293 & 3.3756 & 3.4041 \\
ARMA(3, 0)-GARCH(2, 2) & -1745.653 & 3.3724 & 3.4105 \\
\hline
\end{tabular}




\section{References}

Andersen, T.G, Bollerslev, T., 1998, "Answering the Critics: Yes, ARCH models Do Provide Good Volatility forecast”. International Economic Review, 39, 885-905.

Baillie, R., and Bollerslev, T., 1989, "The Message in Daily Exchange Rates: A Conditional-Variance Tale". Journal of Business and Economic Statistics, 7, 297-305.

Bollerslev, T., 1986, "Generalized Autoregressive Conditional Heteroscedasticity”. Journal of Econometrics, 31, 307-327.

Bollerslev, T., 1987, "A Conditionally Heteroscedastic Time Series Models for Speculative Prices and Rates of Return”. Review of Economics and Statistics, 69, 512-547.

Bollerslev, T., and Wooldridge, J., 1992, "Qausi-Maximum Likelihood Estimation Inference in Dynamic Models with Time-varying Covariance". Econometric Theory, 11, 113-172.

Bollerslev, T. and Ghysels, 1996, "Periodic Autoregressive Conditional Heteroscedasticity". Journal of Business and Economic Statistics, 14, 139-316.

Bollerslev, T., Engle R.F., and Nelson, D., 1994, “ARCH Models". In Handbook of Econometrics Vol. IV, Ed. R. F. Engle and McFadden (Amsterdam North-Holland) pp 2959-3038.

Brailsford, T. and Faff R., 1996, "An Evaluation of Volatility Forecasting Techniques'. Journal of Banking and Finance, 20, 119-138.

Ding, T., Granger, C. W.J., and Engle, R. F., 1993, “A Long Memory Property of Stock Market Returns and a New Model”. Journal of Empirical Finance, 1, 83-106

Engle, R., 1982, "Autoregressive Conditional Heteroscedasticity with Estimates of the Variance of United Kingdom Inflation”. Econometrica, 50, 9871007.

Engle, R., and G. Gonzalez-Rivera, 1991, "Semiparametric ARCH Model". Journal of Business and Economic Statistics, 9, 345-360. 
Glosten, L., Jagnannathan, R., and Runkle, D., 1993, "On the Relation between Expected Return on Stocks”. Journal of Finance, 18, 17791801 .

Hansen, P. R., Lunde, A. and Nason, J. M., 2003a, "Model Confidence Sets for Forecasting Models”, Mimeo, Brown University.

Hansen, P. R., Lunde, A. and Nason, J. M., 2003b, "Choosing the Best Volatility Models: The Model Confidence Set Approach”. Oxford Bulletin of Economics and Statistics, 65, Supplement 0305-9049.

Hsieh, D., 1989, "Modeling Heteroscedasticity in Daily Foreign Exchange Rates". Journal of Business and Economic Statistics, 7, 307-317.

Kaiser, T., 1996, “One Factor GARCH Models for German Stocks -Estimation and Forecasting”. Universitieit Tubingen, Working Paper.

Klaassen, F., 2002, "Improving GARCH Volatility Forecasts". Empirical Economics, 27, 363-394.

Lambert, P., and Laurent, S., 2001, "Modeling Financial Time Series using GARCH-type Models and a Skewed Student Density”. Mimeo, Universiti de Liege.

Lopez, J. A., 2001, "Evaluating the predictive Accuracy of volatility Models". Journal of Forecasting 20(1), 87-109.

Loudon, G., Watt, W., and Yadav, P., 2000, "An Empirical Analysis of Alternative Parametric ARCH Models”. Journal of Applied Econometrics, 15, 117-136.

Marcucci, J., 2005, "Forecasting Stock Market Volatility with RegimeSwitching GARCH Models". Department of Economics, University of California, at San Diego.

Merton, R. C., 1980, “On Estimating the Expected Return on the Market: An Explanatory Investigation”. Journal of Financial Economics 8, 232361.

Mincer, J., and Zarnowitz V., 1969, "The Evaluation of Economic Forecasts and Expectations”. J. Mincer, New York: National Bureau of Economic Research. 
Nelson, D., 1991, "Conditional Heteroscedasticity in Asset Returns: A New Approach”. Econometrica, 59, 319-370.

Pagan, A., and Schwert G., 1990, "Alternative Models for Conditional Stock Volatility”. Journal of Econometrics, 15, 267- 290.

Peters, J., 2001, "Estimating and Forecasting volatility of Stock Indices using Asymmetric GARCH Models”. Mimeo, Universite de Liege.

Schwert, G.W., 1989, "Why does Stock Market Volatility Change over Time? Journal of Finance, 44, 1115-1153.

Sentana, E., 1995, "Quadratic ARCH Models”. Review of Economic Studies, 62, 639-661

Zakoian, J. M., 1994, “Threshold Heteroscedastic Models”. Journal of Economic Dynamics and Control, 18, 931-955. 